\title{
JÜDISCHES \\ UND HEIDNISCHES \\ IM CHRISTLICHEN KULT
}

\author{
EINE VORLESUNG \\ VON \\ GERHARD LOESCHCKE
}

$=\square$

BONN

A. MARCUS UND E. WEBER'S VERLAG

1910 
